\title{
RESULTADOS INSTRUMENTALES OBTENIDOS PARA EL EDIFICIO AISLADO DE LA UTN. EFICIENCIA DE MODELOS NUMÉRICOS PARA PREDECIR LAS RESPUESTAS SÍSMICAS
}

\author{
INSTRUMENTAL RESULTS OBTAINED FOR THE ISOLATED BUILDING OF \\ THE UTN. EFFICIENCY OF NUMERICAL MODELS TO PREDICT SEISMIC \\ RESPONSES
}

\author{
MIGUEL TORNELLO', CARLOS FRAU ${ }^{2}$, ROBERTO AGUIAR ${ }^{3}$, GUSTAVO FOZZATTI ${ }^{4}$ \\ 1 Universidad tecnológica Nacional. Argentino. mtornello@frm.utm.edu.ar \\ 2 Universidad tecnológica Nacional. Argentino. cfrau@frm.utm.edu.ar \\ 3 Escuela politecnica del Ejercito. Ecuador. raguiar@.espe.edu.ec \\ 4 Universidad tecnológica Nacional. Argentino.gfozzatti@frm.utm.edu.ar
}

RESUMEN

Los terremotos son uno de los eventos naturales que han causado la mayor cantidad de pérdidas humanas y económicas en distintas regiones sísmicas del mundo. En las últimas dos décadas, la ingeniería sísmica ha desarrollado nuevas estrategias para controlar y reducir el daño de las construcciones frente a terremotos. Las técnicas más utilizadas se basan en el empleo de dispositivos de disipación de energía y de aislamiento sísmico de base. Si bien ambas estrategias son muy utilizadas en algunos países, fundamentalmente los desarrollados, aún tienen escasas aplicaciones en las zonas sísmicas de Argentina. En el presente trabajo se sintetizan las respuestas sísmicas medidas en términos de aceleraciones, velocidades y desplazamientos de un edificio aislado de tres niveles de la Facultad Regional Mendoza de la Universidad Tecnología Nacional, perteneciente a la residencia de estudiantes de la institución. Los resultados corresponden a un periodo de diez años a partir del momento en que se registró el primer movimiento sísmico sensible en la región, 2005. El proyecto cuenta con instrumental sísmico para monitorear el edificio aislado y otro edificio similar con fundaciones tradicionales. La instrumentación permite comparar las respuestas del edificio aislado con el de base fija, dado que ambos edificios poseen las mismas características arquitectónicas y estructurales y se encuentran separados por escasos metros. El trabajo también muestra el desarrollo de dos modelos numéricos con distinto grado de complejidad matemática que permiten simular el comportamiento del edificio, compararlos con los registros obtenidos del instrumental sísmico y predecir su respuesta frente a la ocurrencia de sismos frecuentes en la región. Los resultados relacionados con la comparación entre el edificio aislado y el mismo edificio pero de base fija, muestran la eficiencia de los dispositivos de aislamiento sísmico instalados y la conveniencia del uso de esta estrategia en construcciones emplazadas en regiones con elevado peligro sísmico. Las conclusiones relacionadas con la eficiencia de los modelos numéricos para predecir la respuesta sísmica del edificio aislado arrojan resultados de aceptable aproximación con discretas discrepancias respecto de la medición instrumental.

PALABRAS CLAVE: protección sísmica, aislamiento sísmico, instrumental sísmico, registros sísmicos, modelos numéricos.
ABSTRACT

Earthquakes are one of the natural events that have caused the greatest amount of human and economic loss in different seismic regions of the world. In the last two decades, seismic engineering has developed new strategies to control and reduce earthquake damage. The most used techniques are based on the use of energy dissipation devices and basic seismic isolation. Although both strategies are widely used in some countries, mainly the developed ones, they still have few applications in the seismic zones of Argentina. In the present work the measured seismic responses in terms of accelerations, velocities and displacements of an isolated building of three levels of the Regional Faculty Mendoza of the National Technology University, pertaining to the student residence of the institution, are synthesized. The results correspond to a period of ten years from the time of the first sensitive seismic movement in the region, 2005. The project has seismic instruments to monitor the isolated building and another similar building with traditional foundations. The instrumentation allows to compare the answers of the isolated building with the fixed base, since both buildings have the same architectural and structural characteristics and are separated by few meters. The work also shows the development of two numerical models with different degrees of mathematical complexity to simulate the behavior of the building, to compare them with the records obtained from the seismic instruments and to predict their response to the occurrence of frequent earthquakes in the region. The results related to the comparison between the isolated building and the same building but with fixed base show the efficiency of the seismic insulation devices installed and the convenience of using this strategy in constructions located in regions with high seismic danger. The conclusions related to the efficiency of the numerical models to predict the seismic response of the isolated building yield results of an acceptable approximation with discrete discrepancies with respect to the instrumental measurement.

KEYWORDS: seismic protection, seismic isolation, seismic instruments, seismic records, numerical models. 


\section{INTRODUCCIÓN}

Aproximadamente un tercio de la población mundial habita en zonas expuestas al riesgo sísmico y vive en edificios con escasas prescripciones sísmicas o bien, diseñados con códigos antiguos y obsoletos (Martelli A., 2005). Los terremotos son fenómenos recurrentes pero fuertemente irregulares en cuanto al tiempo de ocurrencia. Precisamente dicho aspecto incide de manera negativa, en ciertas regiones sísmicas, sobre la conciencia de las personas en general, y de los gobiernos en particular, quienes tienen la responsabilidad de asignar recursos económicos para reducir las vulnerabilidades de las obras, principalmente las esenciales, es decir aquellas que son utilizadas para atender la emergencia posterremoto.

Las construcciones tradicionales diseñadas con códigos modernos pueden considerarse como un progreso significativo dentro de la ingeniería, sin embargo, las mismas siguen presentando limitaciones frente a terremotos destructivos. Precisamente por esta causa es que la atención de muchas investigaciones se ha centrado, en las últimas dos o tres décadas, en poner a punto tecnologías innovadoras para reducir los efectos de los terremotos. Estas nuevas tecnologías, en general, persiguen dos objetivos, el primero superar las limitaciones de una construcción tradicional (admitir daños considerables frente a fuertes terremotos, incluida la inutilización del edificio, aún construidos con códigos modernos) y el segundo asistir las construcciones existentes con escasa o ninguna prescripción sísmica.

Las estrategias de protección sísmica más utilizadas y difundidas a nivel mundial son el aislamiento de base y los dispositivos de disipación de energía, ambas han mostrado resultados satisfactorios en regiones sísmicas de muy elevada peligrosidad (Martelli A., 2005).

Sobre ambas estrategias existen numerosas aplicaciones a nivel mundial sin embargo, en las zonas sísmicas de Argentina, el uso de la técnica es reducida. Se conoce que la energía sísmica se transmite a la estructura a través de la fundación, por lo tanto, el principio general del aislamiento sísmico es desacoplar la construcción del movimiento del terreno. Dicho aislamiento se realiza usualmente insertando, entre la construcción y la fundación, dispositivos de apoyo con baja rigidez horizontal y/o vertical que permiten liberar los desplazamientos del edificio como consecuencia del terremoto (Tornello M. y Sarrazin M., 2007). Los disposi- tivos son diseñados con una rigidez adecuada frente a acciones frecuentes, por ejemplo la acción del viento, de tal manera de no provocar sobre la estructura desplazamientos sensibles.

El nivel de seguridad que se consigue con los sistemas de aislamiento sísmico es mayor que el logrado por una construcción sismoresistente convencional, aun para los terremotos que causan graves daños en las construcciones y el colapso de aquellas con escasas prescripciones sísmicas (Tornello M. et al, 2013).

Basado en esa línea de pensamiento, en 2002 se abordaron en la Facultad Regional Mendoza de la UTN, proyectos de investigación relacionados con la protección sísmica. En tal sentido, uno de los primeros proyectos en el país fue la residencia de estudiantes de la Facultad Regional Mendoza de la Universidad Tecnológica Nacional, la cual es un conjunto de tres torres, cuya construcción fue concluida en el 2005. El complejo lo completa un edificio de dos niveles para administración y aulas de posgrado (Tornello M. y Frau C., 2010). El sector de residencia, perfectamente diferenciado del anterior, se compone de tres edificios de tres niveles cada uno con idénticas características arquitectónicas y estructurales. Esos edificios fueron designados en la etapa de proyecto como Torres 1, 2 y 3 (T1, T2, T3) destinado a un dormitorios para los residentes y visitantes.

La T3 se caracteriza por estar montada sobre un sistema de aislamiento sísmico de base, mientras que la T1 y T2 fueron construidas de manera tradicional es decir, con base fija (Tornello M y Frau C., 2010). A fines 2005 se procedió a la instrumentación sísmica de T2 y T3 las cuales están separadas por escasos metros; situación que ha permitido, desde su concreción, comparar sus respuestas frente a la ocurrencia de sismos menores sentidos en la región ya sea de foco cercano como de epicentros lejanos (Tornello M, et al, 2010).

El presente trabajo muestra los resultados de los sismos más relevantes de los últimos diez años y se comparan las respuestas medidas a través del instrumental sísmico instalado en la T3, con aislamiento sísmico, y en la torre $\mathrm{T} 2$, con base fija. Los resultados obtenidos, en tal sentido, indican la conveniencia del uso del aislamiento sísmico como técnica de protección y además pone de manifiesto el correcto funcionamiento del conjunto sistema de aislamiento - edificio fundamentalmente, en aspectos relacionados con el desacoplamiento de las obras complementarias. También se compara 
la respuesta medida a través del instrumental sísmico instalado en la torre $\mathrm{T} 3$ con aislamiento sísmico y aquella obtenida a partir de modelos numéricos tridimensionales y modelos simples lineales. Los resultados obtenidos para éstos análisis indican una aceptable aproximación entre lo determinado con los modelos de análisis y lo medido por el instrumental sísmico instalado en los edificios.

\section{CARACTERÍSTICAS DEL EDIFICIO AISLADO}

Los tres edificios destinados a dormitorios poseen las mismas características arquitectónicas y estructurales, constan de tres niveles y planta rectangular de $8,0 \times 7,60 \mathrm{~m}$. La superficie de cada torre destinada a dormitorios es de 176 $\mathrm{m} 2$, lo que hace un total de $528 \mathrm{~m} 2$ entre los tres edificios. Todos los cuerpos del complejo fueron construidos con estructura de hormigón armado y mampostería encadenada y armada. Los muros exteriores son de mampostería (ladrillo macizo cocido) con espesores de 20 y $30 \mathrm{~cm}$. Los entrepisos y cubierta de techo están conformados por una losa prefabricada plana y una capa de hormigón armado superior para lograr una estructura monolítica. Las divisiones interiores han sido realizadas con placas de roca de yeso. Las fundaciones han sido resueltas con base corridas y vigas rígidas de fundación incorporadas a dichas bases de hormigón armado. La cota de fundación de las bases corridas se ubica entre -2,00 y -2,50 m (Tornello M. y Sarrazin M., 2007). En la figura 1 se muestra la T3 (edificio aislado) y la T2 (edificio de base fija) en la etapa de construcción y de obra terminada.

El sistema de aislamiento instalado en la T3 permite desplazamientos verticales y horizontales y provee amortiguamiento en las mismas direcciones, a través del trabajo conjunto de los aisladores de resortes de acero y amortiguadores viscosos (Tornello M., 2012). Las masas del edificio con aislamiento sísmico se indican en la tabla 1; los valores indicados en la misma incluyen una participación del $25 \%$ de la carga viva. A los efectos comparativos se indican también los datos de la T2, de base fija. En el edificio aislado, la masa del primer nivel incluye la correspondiente al nivel considerado, más la correspondiente a la losa y vigas inmediatamente por encima del sistema de aislamiento. Las masas correspondientes a los dispositivos de aislamiento se han despreciado debido a su escasa incidencia comparada con la masa del edifico emplazado por encima de los dispositivos de aislamiento.
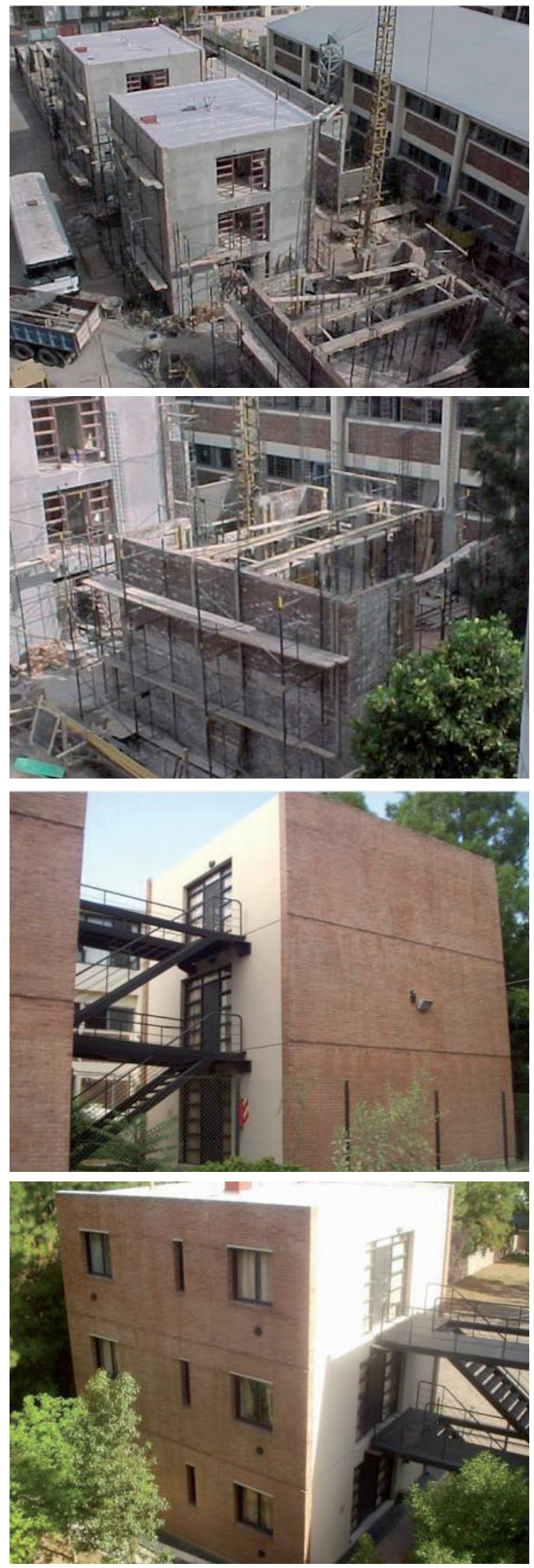

Figura 1. Edificios de dormitorios de la residencia de estudiantes de la UTN (Torres T2 y T3). Etapa de construcción y obra terminadaz. 
TABLA 1. MASAS DEL EDIFICIO AISLADO Y DEL EDIFICIO CON BASE FIJA

\begin{tabular}{rrr}
\hline NIVEL & $\begin{array}{r}\text { EDIFICIO CON BASE FIJA (T2) } \\
\text { (KN. S2/M) }\end{array}$ & $\begin{array}{r}\text { EDIFICIO CON AISLAMIENTO } \\
\text { SÍSMICO (T3) (KN. S2/M) }\end{array}$ \\
\hline 1 & 69.06 & 158.77 \\
\hline 2 & 56.33 & 56.33 \\
\hline 3 & 46.96 & 46.96 \\
total & 172.35 & 262.06 \\
\hline
\end{tabular}

CARACTERÍSTICAS DE LOS DISPOSITIVOS DE AISLAMIENTO SÍSMICO

Los dispositivos de aislamiento fueron provistos por la firma Gerb $^{\circledR}$ fabricados en Alemania. El sistema de aislamiento está compuesto por cuatro paquetes de resortes y cuatro amortiguadores independientes de eje vertical $\left(\right.$ Visco $\left.^{\circledR}\right)$, fabricados por la misma empresa y dispuestos en cada vértice del edificio, debajo de la viga perimetral que soporta la superestructura (Tornello M. y Sarrazin M., 2012).

Los sistemas de resortes para aislamiento sísmico (GERB Control System ${ }^{\circledR}$, GCS) fueron originalmente diseñados para controlar las vibraciones que generaban las turbinas en plantas hidroeléctricas y, posteriormente, extendidos a construcciones civiles (Nawrotzki P., 2001). El aislador está conformado por paquetes de resortes con baja rigidez en sentido horizontal y vertical, sin embargo para aumentar el amortiguamiento del sistema de aislamiento se instalan en paralelo con amortiguadores visco elásticos (Visco ${ }^{\circledR}$ ), (figuras 2a y 2b) (Tornello M. y Sarrazin M., 2012).
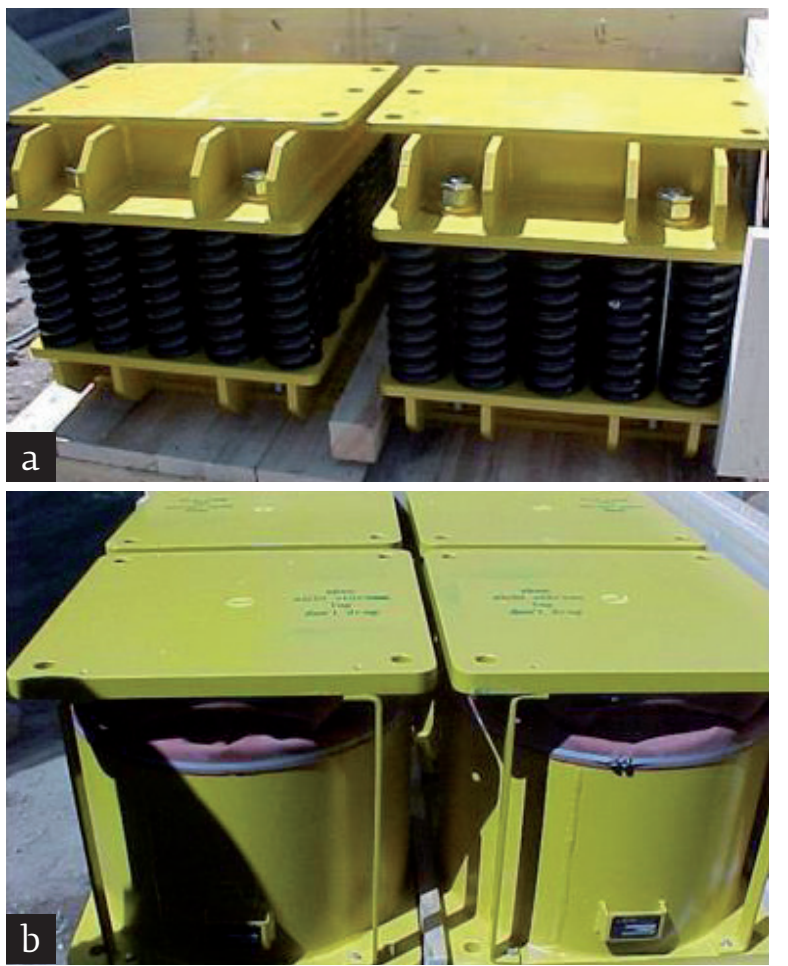

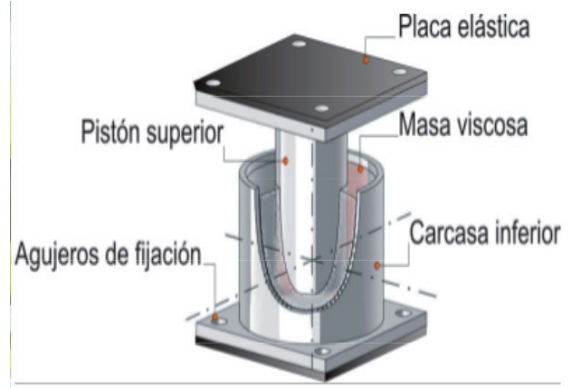

Figura 2. a, b.) Paquete de resortes metálicos $\mathrm{GERB}^{\circledR}$. c) Amortiguadores viscosos GERB (Visco $\left.{ }^{\circledR}\right)$.

La cantidad de resortes que componen el paquete es función de la rigidez vertical, horizontal y de las capacidades a cargas estáticas y dinámicas impuestas por las acciones en servicio y sísmicas. Como consecuencia de una mayor carga vertical en uno de los lados del edificio, dos de los paquetes están compuestos por 30 resortes, con una capacidad de carga vertical de $921 \mathrm{kN}$, y los otros dos por 28 resortes con una capacidad de carga vertical de $768 \mathrm{kN}$ (Tornello M. y Sarrazin M., 2012). En la tabla 2 se resumen las características principales del sistema de aislamiento.

\begin{tabular}{|c|c|c|c|c|}
\hline PARÁMETRO & NOTACIÓN & $\begin{array}{r}28 \\
\text { RESORTES }\end{array}$ & $\begin{array}{r}30 \\
\text { RESORTES }\end{array}$ & UNIDAD \\
\hline $\begin{array}{l}\text { Capacidad nominal carga } \\
\text { vertical }\end{array}$ & $\mathrm{F}_{\mathrm{v}}$ & 768 & 921 & KN \\
\hline Rigidez vertical & $K_{v}$ & 29500 & 35400 & $\mathrm{KN} / \mathrm{m}$ \\
\hline Rigidez horizontal & $K_{h}$ & 3940 & 4730 & $\mathrm{KN} / \mathrm{m}$ \\
\hline Amortiguamiento horizontal & $C_{h}$ & 26 & 26 & $\%$ \\
\hline Amortiguamiento vertical & $C_{v}$ & 13 & 13 & $\%$ \\
\hline $\begin{array}{l}\text { Diámetro exterior del } \\
\text { resorte }\end{array}$ & $D$ & 105 & 105 & $\mathrm{~mm}$ \\
\hline Diámetro espira resorte & $d$ & 26 & 26 & $\mathrm{~mm}$ \\
\hline Altura libre del resorte & $h_{s}$ & 271 & 271 & $\mathrm{~mm}$ \\
\hline
\end{tabular}

En la figura 3 se muestran los dispositivos de aislamiento (aisladores y amortiguadores) en su posición final y debajo del edificio (Torre T3).

La rigidez horizontal y vertical de los resortes son funciones del módulo de corte del material del resorte, diámetro de la espira que conforma el resorte, número de espiras activas, diámetro exterior del resorte y altura libre del resorte (Tornello M., 2012). La rigidez lateral de los resortes, sujetos a acciones dinámicas se encuentra influenciada por un efecto combinado de flexión y torsión (Nawrotzki P., 2001).

El sistema de aislamiento utilizado presenta un fuerte acoplamiento entre el movimiento horizontal y el pendular debido a que el centro 
de gravedad de la estructura aislada se encuentra por encima del centro de rigidez del mismo (figura 4), por lo tanto el sistema resulta útil cuando el centro de gravedad y el centro de rigidez se encuentran en el mismo nivel situación que se presenta con cierta frecuencia (Naeim F. y Kelly J., 1999).
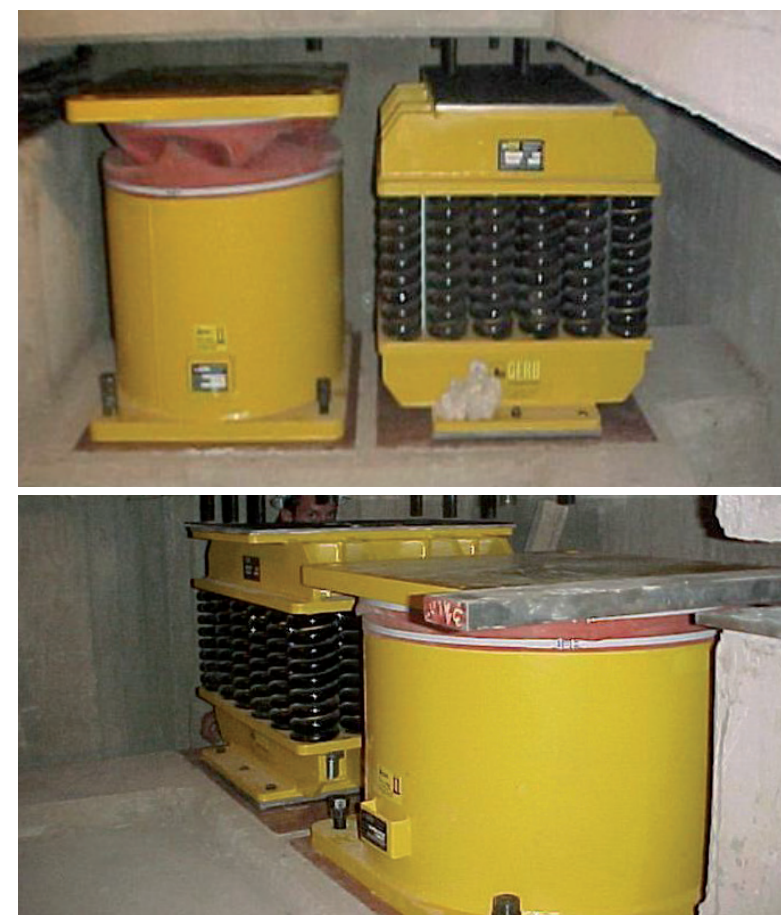

Figura 3.

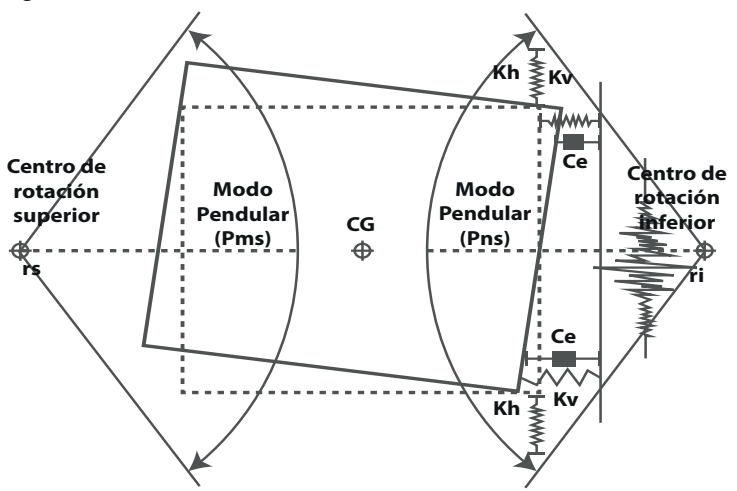

Figura 4. Mecanismo de disipación de energía de los dispositivos GERB ${ }^{\circledR}$. Modos pendulares

\section{INSTRUMENTAL SÍSMICO INSTALADO EN LOS EDIFICIOS}

Los instrumentos instalados son acelerómetros marca Kinemetrics, modelo Altus K2 con un sensor triaxial interno. El mismo actúa como central de adquisición de datos y es el receptor de otros nueve canales externos de registros de aceleración, por lo tanto el sistema instalado tiene capacidad para el registro simultáneo de aceleración, de doce canales (Tornello M., et al, 2010). El emplazamiento de los distintos sensores se resume en la tabla 3 , mientras que en la figura 5 se representa gráficamente la ubicación en el edificio con aislamiento sísmico.

En la Torre 2 que corresponde al edificio de base fija, se encuentra instalado en la $\mathrm{cu}^{-}$ bierta de techo un sensor triaxial que permite el registro sísmico de las tres componentes del movimiento. La T2 se emplaza a escasos metros del edificio aislado por lo tanto los valores registrados en dicho edificio permiten la comparación de los resultados obtenidos en las componentes 8 y 9 del edificio con aislamiento sísmico. En la figura 6 se muestran algunos de los sensores sísmicos instalados en los distintos niveles del edificio junto a sus protecciones metálicas.

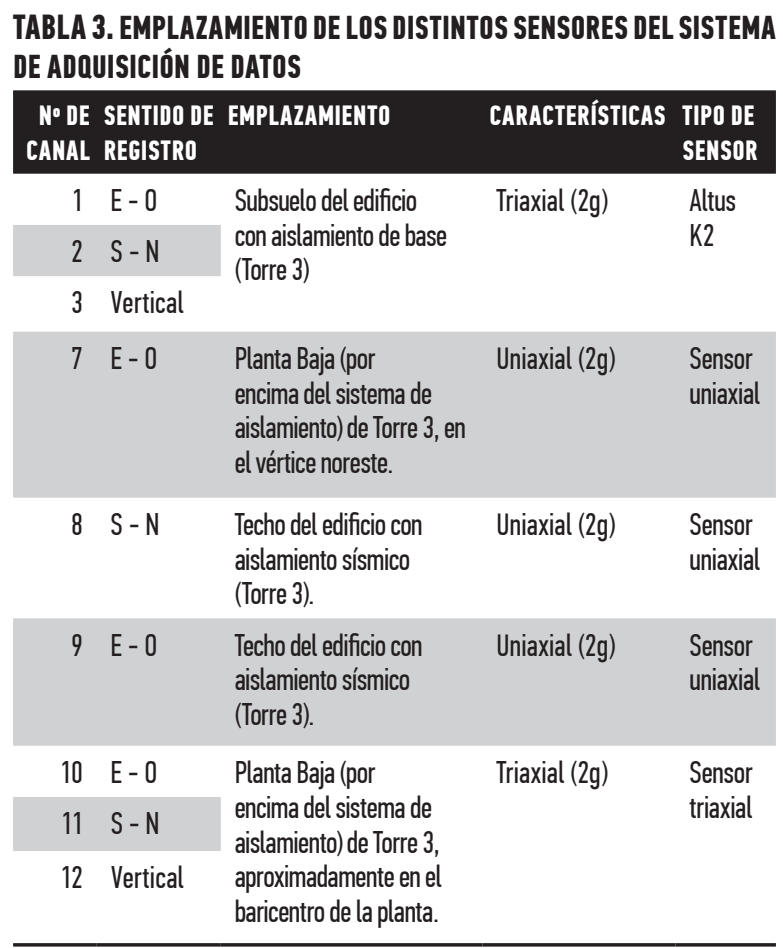

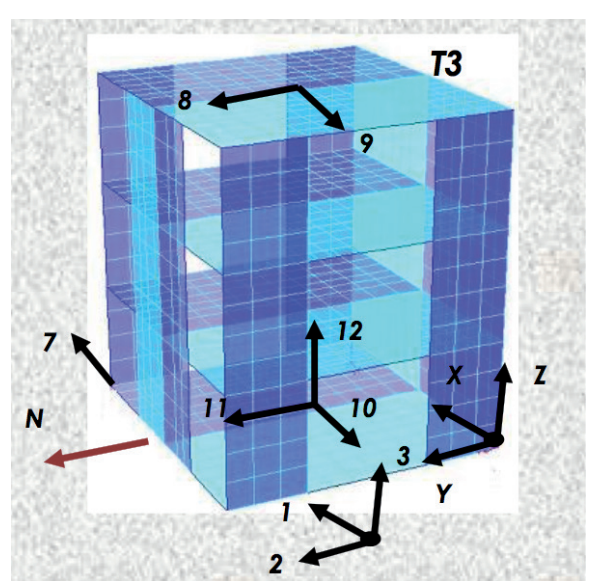

Figura 5. Posición esquemática del instrumental instalado en la T3 y dirección de los registros. 

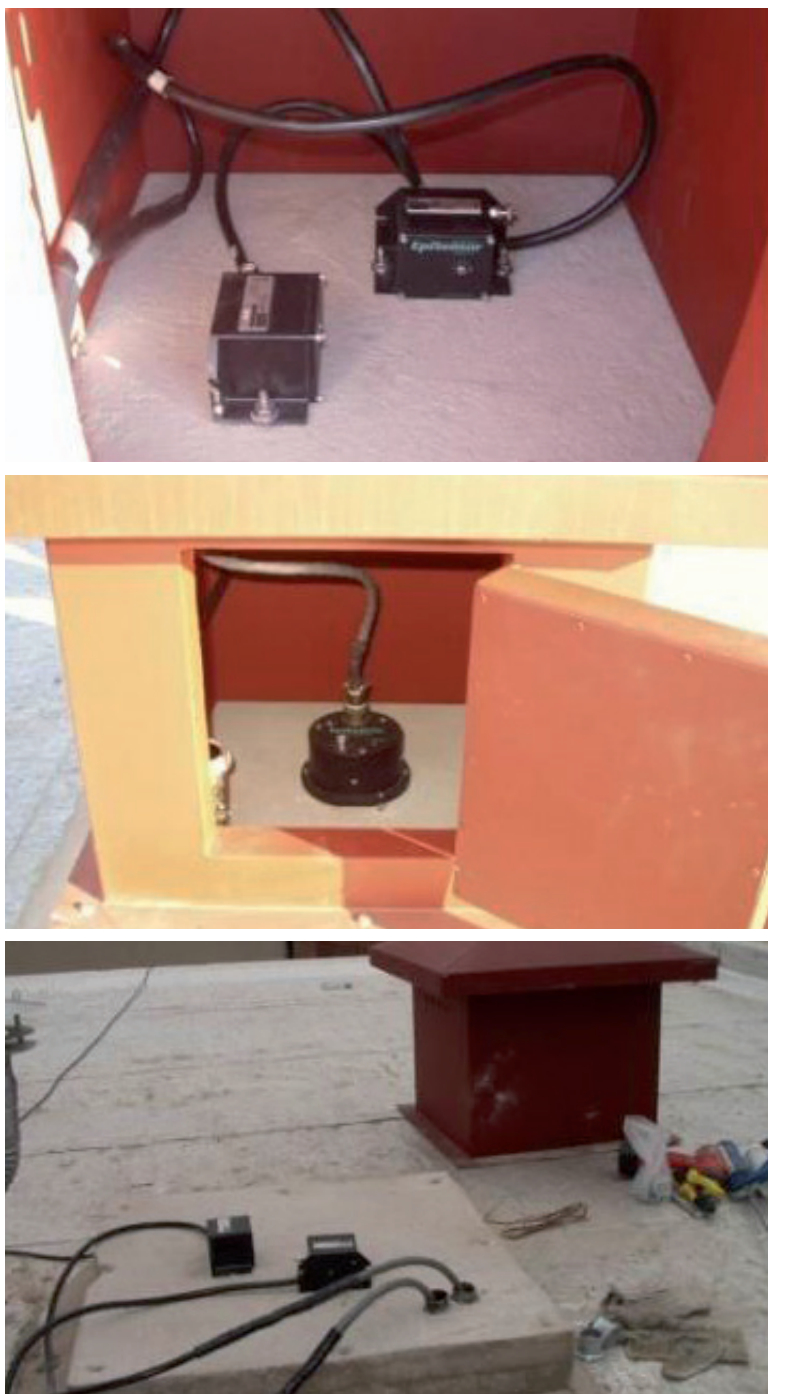

Figura 6. Sensores sísmicos instalados en el edificio aislado y en el edificio con base fija.

RESPUESTAS MEDIDAS EN LOS EDIFICIOS

El instrumental instalado en los edificios de la residencia estudiantil, registró, por primera vez, el evento sísmico del 09/09/2005, ocurrido a las 08:25:26 hs, con epicentro a $170 \mathrm{Km}$ de la ciudad de Mendoza, magnitud estimada en 5.2 en 1 a escala de Richter e Intensidad Mercalli Modificada entre IV y V. Desde la fecha citada, el instrumental ha continuado registrando los eventos sísmicos sentidos en la región de manera ininterrumpida y hoy se cuenta con una base de datos de más cien eventos sísmicos registrados.

Con el objeto de evaluar el comportamiento al edificio aislado respecto del de base fija, se comparan los respectivos registros de aceleraciones. En la figura 7 se muestran los resultados obtenidos para algunos los principales eventos sísmicos registrados por el instrumental. Por razones de extensión, se muestra sólo los resultados de un registro por año. Los gráficos muestran la com- paración de las aceleraciones y el desplazamiento para el mismo evento sísmico, del edifico aislado. Con relación a las aceleraciones (gráficos de la izquierda) en el eje horizontal se ha representado la aceleración normalizada respecto al valor registrado a nivel del techo del edificio aislado y en el eje vertical se indica el nivel del edificio. Con relación a los desplazamientos (gráficos de la derecha) en el eje horizontal se han representado los desplazamientos normalizados respecto del valor registrado en el nivel del techo del edificio aislado y en el eje vertical se indica el nivel del edificio.
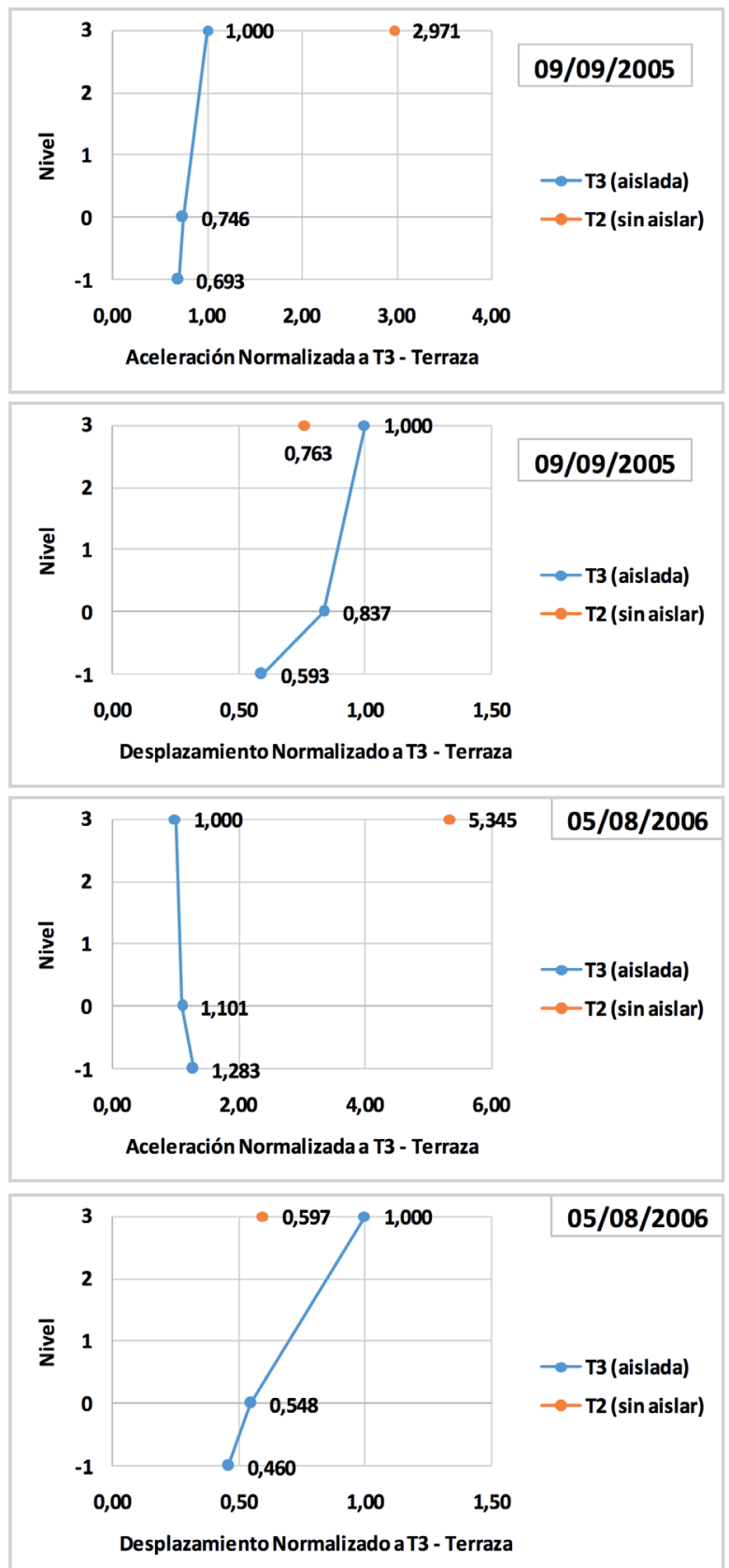

Figura 7. Comparación de las aceleraciones del edificio aislado con el edificio de base fija (gráficos de la izquierda). Desplazamientos registrados del edificio con aislamiento sísmico (gráficos de la derecha) para los sismos del 9 de septiembre de 2005 y 5 de agosto de 2006. 

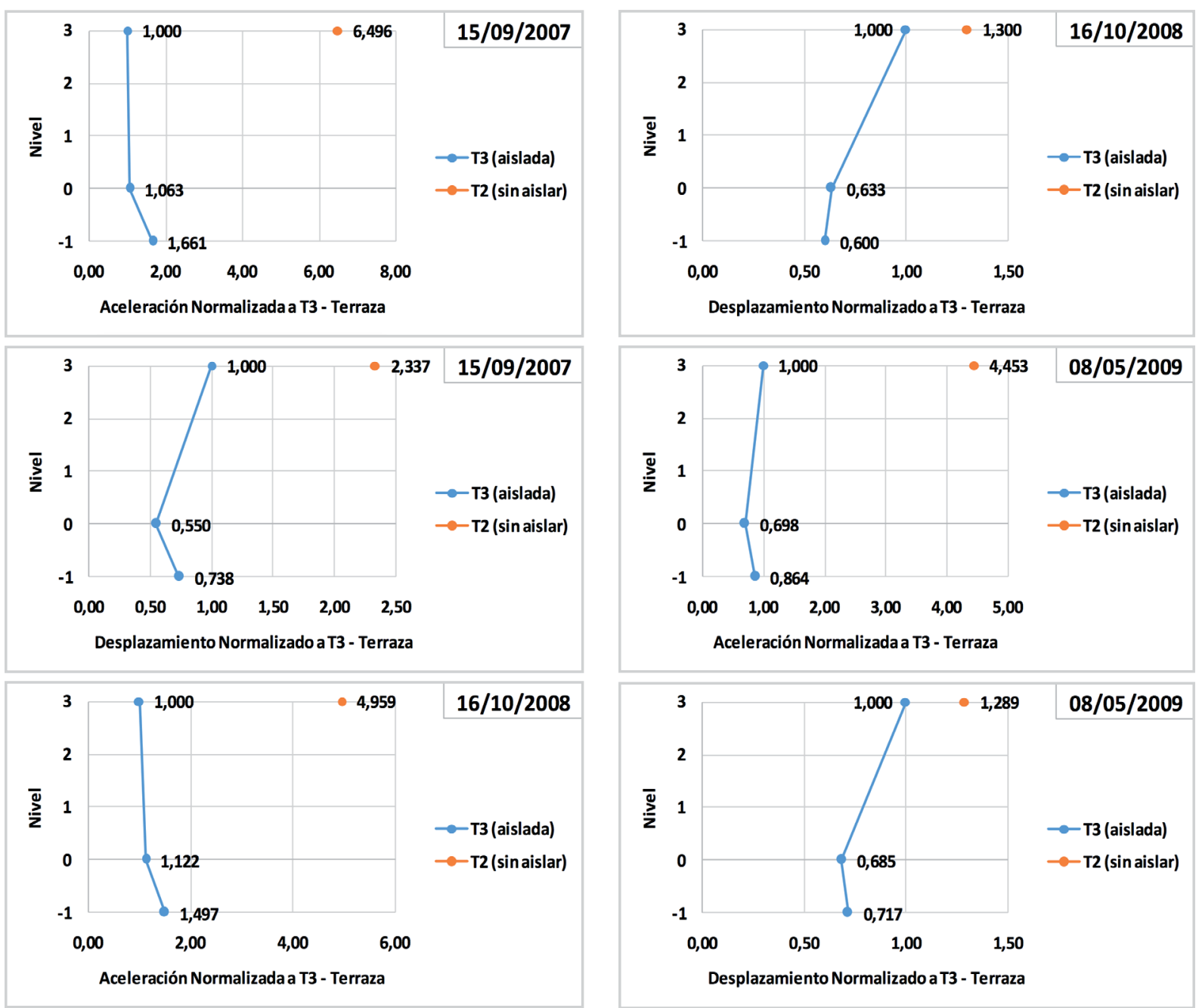

Figura 8. Comparación de las aceleraciones del edificio aislado con el edificio de base fija (gráficos de la izquierda). Desplazamientos registrados del edificio con aislamiento sísmico (gráficos de la derecha).
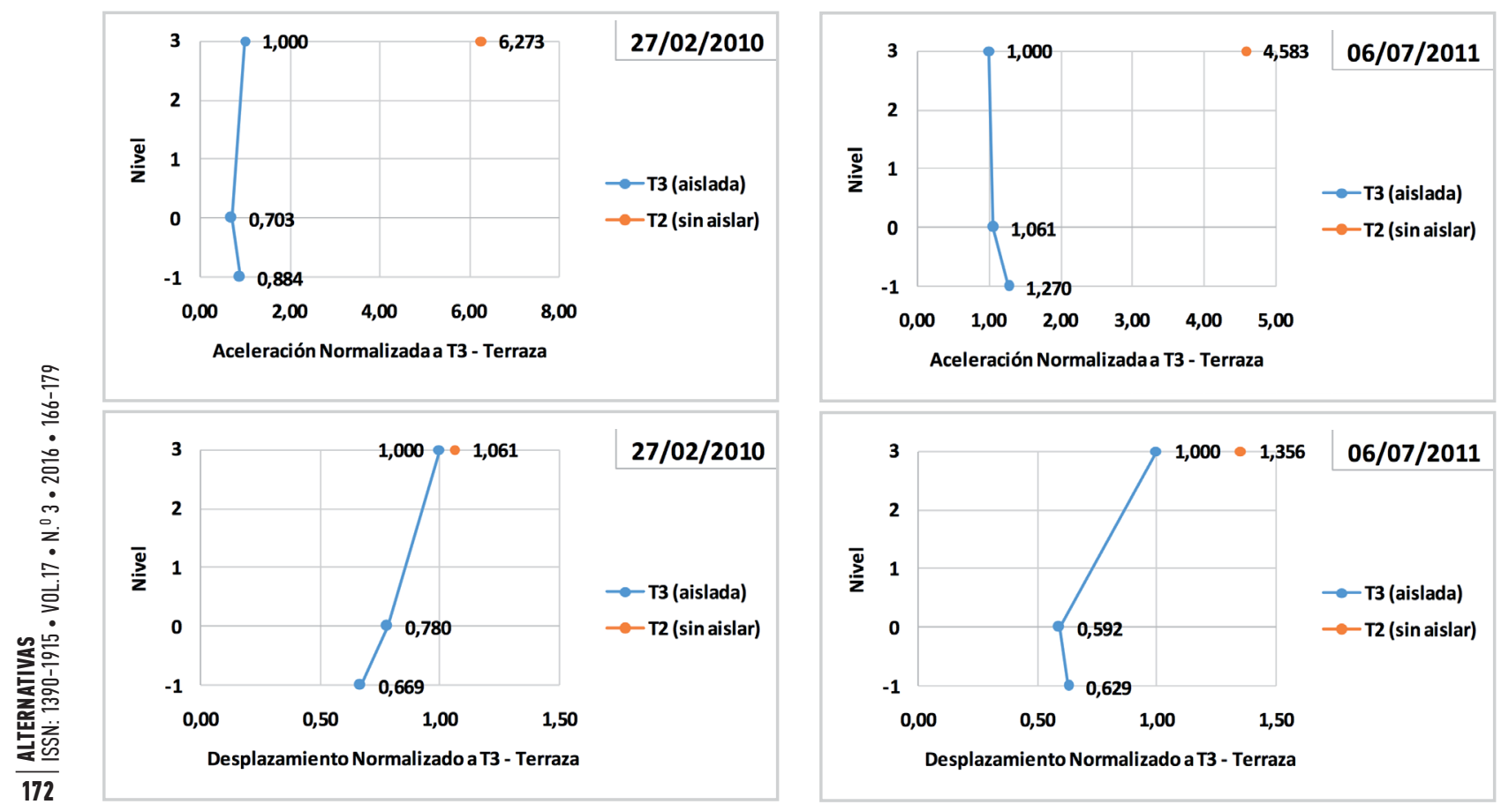

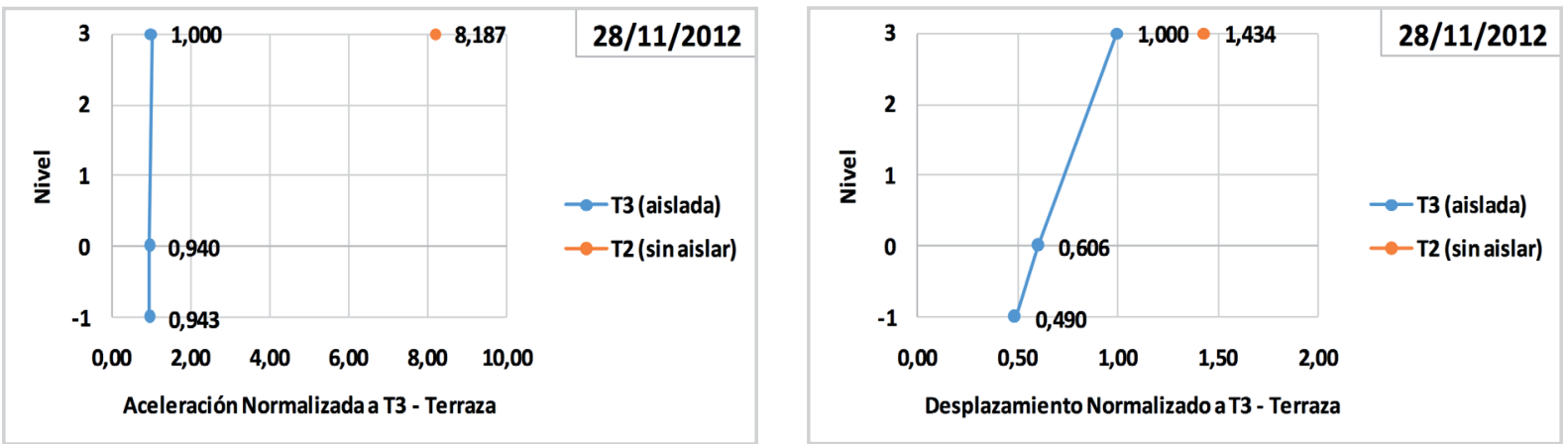

Figura 9. Comparación de las aceleraciones del edificio aislado con el edificio de base fija (gráficos de la izquierda). Desplazamientos registrados del edificio con aislamiento sísmico (gráficos de la derecha).
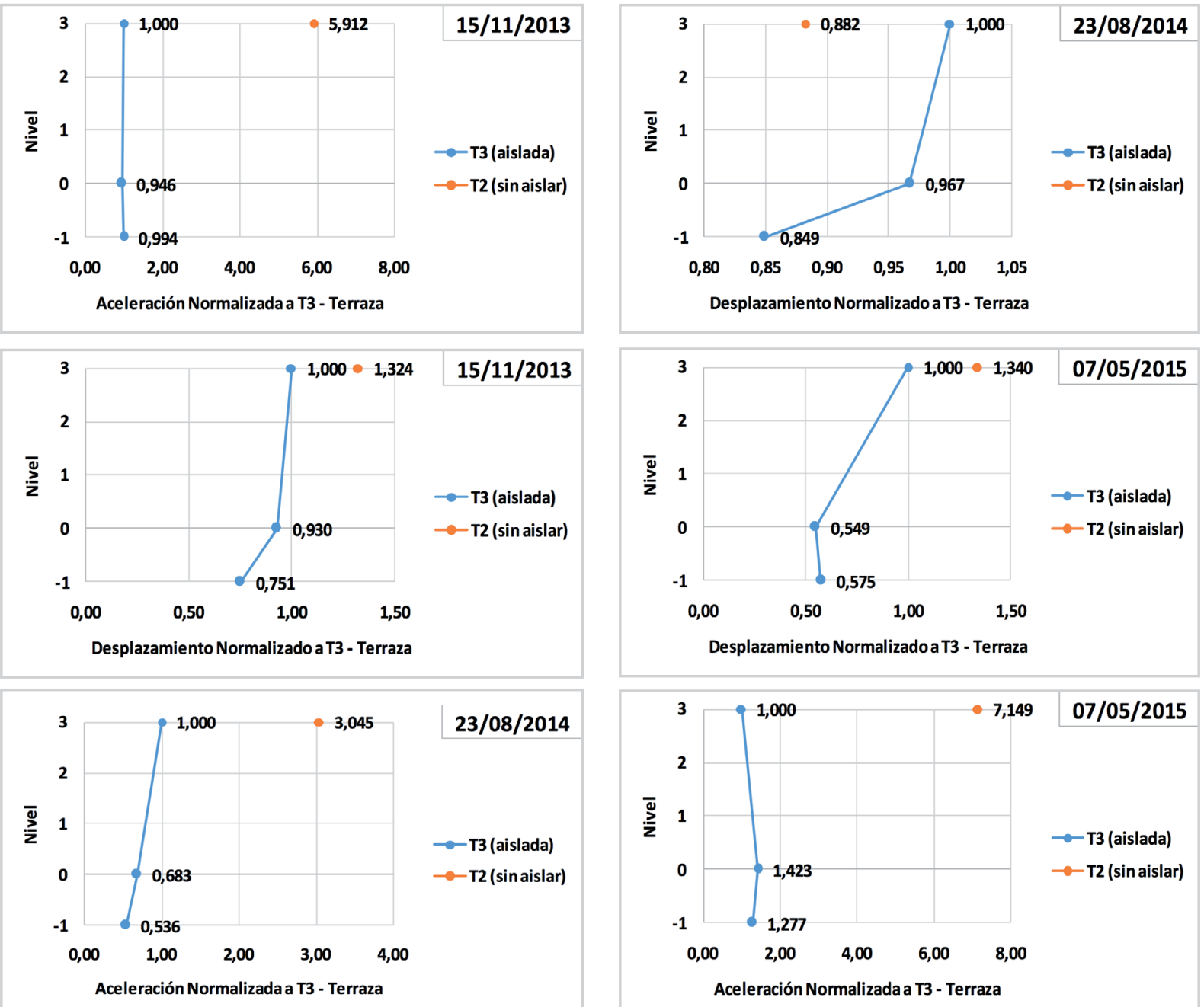

Figura 10. Comparación de las aceleraciones del edificio aislado con el edificio de base fija (gráficos de la izquierda). Desplazamientos registrados del edificio con aislamiento sísmico (gráficos de la derecha).

MODELOS NUMÉRICOS MODELO TRIDIMENSIONAL NO LINEAL (MTNL) Las respuestas dinámicas analíticas han sido evaluadas mediante un modelo 3D de elementos finitos y mediante un análisis no lineal en el dominio del tiempo (time history). La no linealidad ha sido concentrada solamente en los dispositivos de aislamiento (Jangid N. y Kelly J.,
2001). Se han evaluado respuestas en términos de aceleraciones velocidades y desplazamientos inmediatamente por encima y por debajo de los dispositivos de aislamiento; esto es a nivel de planta baja y bases. En la figura 8 se muestra el modelo en elementos finitos utilizado para comparar las respuestas teóricas con las realmente medidas del instrumental instalado. 
Los dispositivos de aislamiento fueron modelados utilizando el elemento tipo "LinkSupport". Se definieron, para cada dirección de análisis, horizontal y vertical, las rigideces efectivas y el coeficiente de amortiguamiento de los dispositivos de aislamiento. El modelo utilizado obtiene la respuesta empleando la relación $f=k d_{K}=c \dot{d}_{c}^{c \exp }$, donde $k$ es la rigidez de los aisladores, $c$ es el coeficiente de amortiguamiento, $d k$ es el desplazamiento de los aisladores y $\dot{d}_{c}$ es la velocidad del amortiguador (Wilson E., 2002).

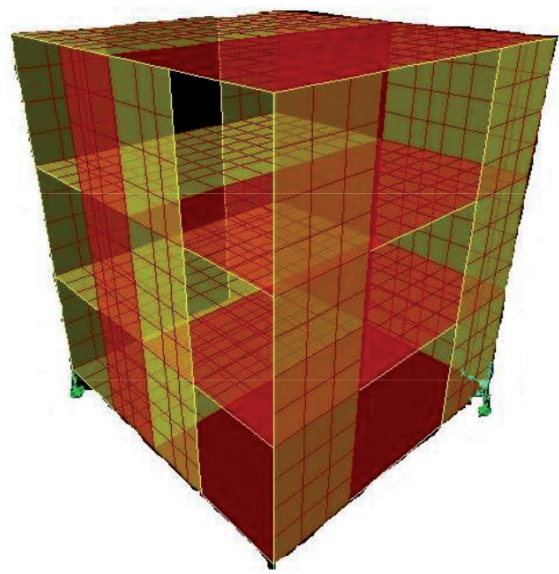

Figura 11. Modelo 3D de elementos Finitos utilizado para evaluar la respuesta analítica del edificio aislado.

Para encontrar las respuestas en términos de aceleraciones, velocidades y desplazamiento se ha utilizado el método de la integración paso a paso de las ecuaciones de movimiento que permite obtener el vector de coordenadas generalizadas (ecuación 1) y su derivada primera y segunda $([\dot{\eta}],[\ddot{\eta}])$, las cuales tienen la misma forma que le ecuación (1).

$$
[\eta]=\left[\begin{array}{lll}
\eta_{11} & \eta_{1 i} & \eta_{1 m} \\
\eta_{21} & \eta_{2 i} & \eta_{1 m} \\
\eta_{31} & \eta_{3 i} & \eta_{1 m}
\end{array}\right]
$$

Los valores numéricos de las respuestas, por ejemplo en términos de desplazamiento fueron determinados con la expresión (2).

$$
[X]=\{\phi\}[\eta]
$$

Donde $\{\phi\}$ son las formas modales y representan los valores propios de las ecuaciones de equilibrio dinámico. Conocidos los desplazamientos puede determinarse las respuestas en términos de velocidades y aceleraciones (ecuación 3 y 4, respectivamente).

$$
[\dot{X}]=\{\phi\}[\dot{\eta}]
$$

$$
[\ddot{X}]=\{\phi\}[\ddot{\eta}]
$$

Para ajustar el modelo aislado GCS, a lo registrado por el instrumental sísmico instalado, se ha tenido en cuenta la dependencia de la rigidez y amortiguamiento al desplazamiento y a la velocidad sin embargo, tanto la rigidez como el amortiguamiento se han considerado constantes. Además y debido a la presencia de los amortiguadores en la base del edificio, el amortiguamiento del modelo ha sido considerado como no proporcional. Estudios previos indican que los dispositivos de aislamiento disipan energía a través de modos pendulares (figura 4) mientras que la superestructura permanece en rango elástico y se mueve como un cuerpo rígido debido a la baja rigidez vertical y horizontal de los paquetes de resortes (Aguiar Falconi R, y Tornello M., 2010).

\section{MODELO SIMPLE LINEAL (MSL)}

Adicionalmente se obtuvo la repuesta en el tiempo para un sistema lineal simple de un grado de libertad, analizando cada dirección horizontal en forma independiente. Las características dinámicas se obtuvieron a partir de la masa del edificio por encima del sistema de aislamiento considerado infinitamente rígido y la rigidez horizontal dada exclusivamente por el sistema de aislamiento. Así, para una razón de amortiguamiento del $26 \%$ resultó un periodo amortiguado de 0.80 segundos, idéntica en ambas direcciones horizontales. La historia de respuestas se realizó mediante la integración numérica de la ecuación general de la dinámica por el método de Newmark, ecuación 5, (Chopra, 2014; Frau y Silva, 2003).

$$
m \ddot{u}+c \ddot{u}+k \ddot{u}=-m \ddot{u}_{g}
$$

Donde $m$ es la masa des sistema, $c$ el coeficiente de amortiguamiento viscoso y $k$ la rigidez del sistema; en tanto que $u$, representa el desplazamiento relativo de la masa indicando con uno o dos puntos la derivada primera o segunda respecto del tiempo.

\section{RESULTADOS OBTENIDOS Y VALORES MEDIDOS}

A partir del registro de aceleraciones en el tiempo medidas por los acelerómetros instalados en el edificio aislado, se realizó una doble integración numérica para obtener las velocidades y desplazamientos. El procesamiento de los registros se realizó con el software SMA desarrollado por la compañía Kinemetrics Inc. 
fabricante de los acelerómetros instalados ( $\mathrm{Ki}^{-}$ nemetric, 2002). La tabla 4 resume los valores máximos encontrados para cada uno de los sismos estudiados en la base y en la planta baja, es decir inmediatamente por debajo y por encima del sistema de aislamiento.

TABLA 4. VALORES MÁXIMOS MEDIDOS EN BASE Y PLANTA BAJA

\begin{tabular}{|c|c|c|c|c|c|c|c|}
\hline \multirow{3}{*}{\multicolumn{2}{|c|}{ FECHA }} & SUBSUEL & & \multicolumn{4}{|c|}{ PLANTA BAJA } \\
\hline & & \multicolumn{3}{|c|}{ Canal 1E-W } & \multicolumn{3}{|c|}{ Canal $2 \mathrm{~N}-\mathrm{S}$} \\
\hline & & $\begin{array}{r}\text { PGA } \\
\text { [cm/s2] }\end{array}$ & $\begin{array}{r}\mathrm{PGV} \\
{[\mathrm{cm} / \mathrm{s}]}\end{array}$ & $\begin{array}{l}\text { PGD } \\
\text { [cm] }\end{array}$ & $\begin{array}{r}\text { PGA } \\
{[\mathrm{cm} / \mathrm{s} 2]}\end{array}$ & $\begin{array}{r}\mathrm{PGV} \\
{[\mathrm{cm} / \mathrm{s}]}\end{array}$ & $\begin{array}{l}\text { PGD } \\
{[\mathrm{cm}]}\end{array}$ \\
\hline \multirow{2}{*}{$\begin{array}{l}05 / 08 / \\
2006\end{array}$} & Parametro & $-122,14$ & $-3,847$ & 0,392 & 94,69 & 3,28 & 0,433 \\
\hline & Tiempo [s] & 25,695 & 25,72 & 25,68 & 26,06 & 24,86 & 25,03 \\
\hline \multirow{2}{*}{$\begin{array}{l}\text { 15/09/ } \\
2007\end{array}$} & Parametro & 54,446 & 1,131 & $-0,043$ & 41,425 & 1,324 & $-0,057$ \\
\hline & Tiempo [s] & 23,43 & 23,475 & 23,44 & 23,445 & 23,485 & 23,44 \\
\hline \multirow{2}{*}{$\begin{array}{l}16 / 10 / \\
2008\end{array}$} & Parametro & 44,562 & $-1,452$ & 0,054 & $-22,087$ & 0,487 & 0,036 \\
\hline & Tiempo [s] & 24,365 & 24,11 & 24,065 & 24,37 & 24,01 & 24,06 \\
\hline \multirow{2}{*}{$\begin{array}{l}27 / 02 / \\
2010\end{array}$} & Parametro & $-15,92$ & $-7,687$ & 4,362 & $-13,288$ & 6,968 & $-4,154$ \\
\hline & Tiempo [s] & 94,825 & 95,865 & 94,825 & 110,285 & 88,54 & 104,34 \\
\hline
\end{tabular}

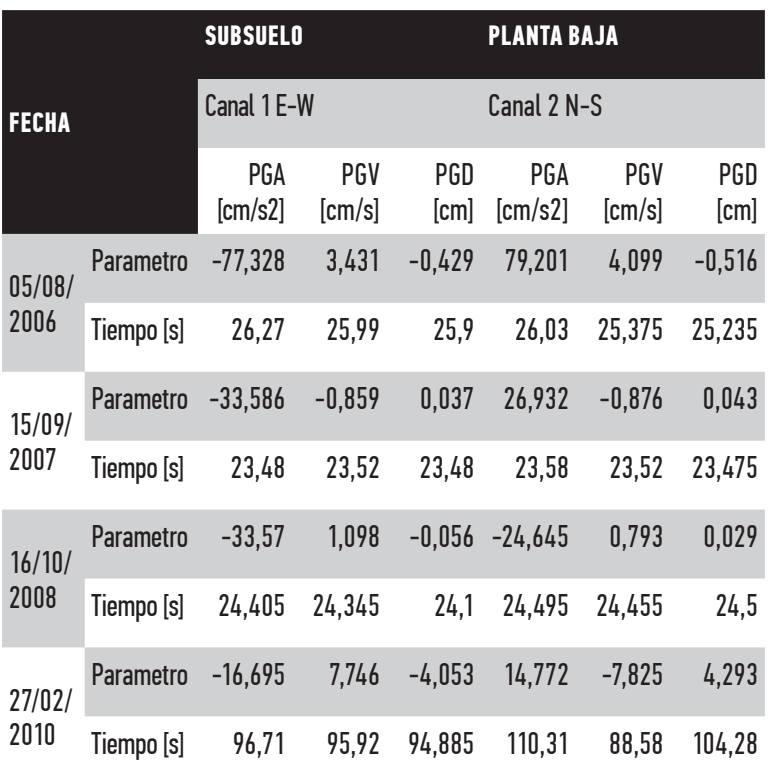

En las figuras 9 a 14 se representan para cada sismo y para cada una de las componentes horizontales la aceleración, velocidad y desplazamiento correspondientes a los valores registrados a nivel de base (línea azul) y los correspondientes a nivel de planta baja, es decir inmediatamente por encima del sistema de aislamiento (línea verde); la representación se limita a la ventana de tiempo principal de cada sismo.
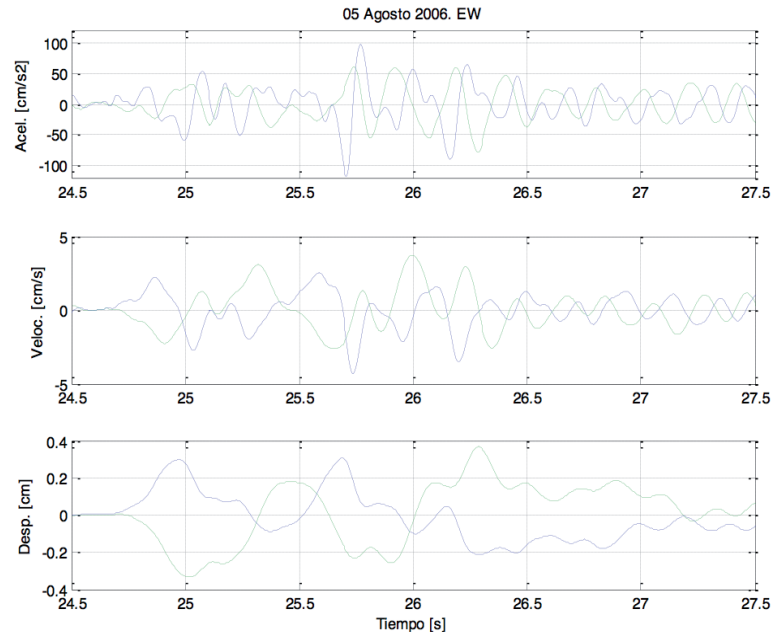

Figura 12. Aceleraciones, velocidades y desplazamientos, registradas a nivel de base (azul) y por encima del sistema de aislamiento (verde). Componente E-W, sismo del 6 Agosto 2006.
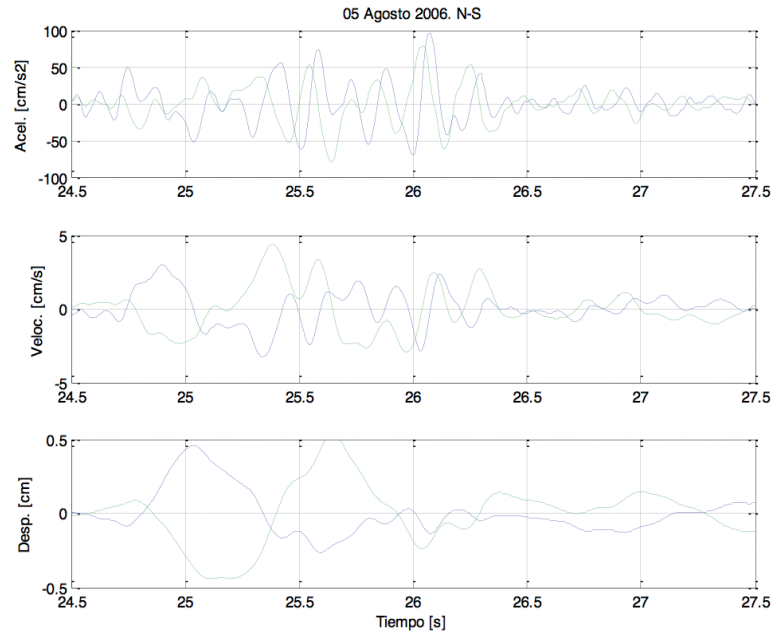

Figura 13. Aceleraciones, velocidades y desplazamientos, registradas a nivel de base (azul) y por encima del sistema de aislamiento (verde). Componente N-S, sismo del 6 Agosto 2006.
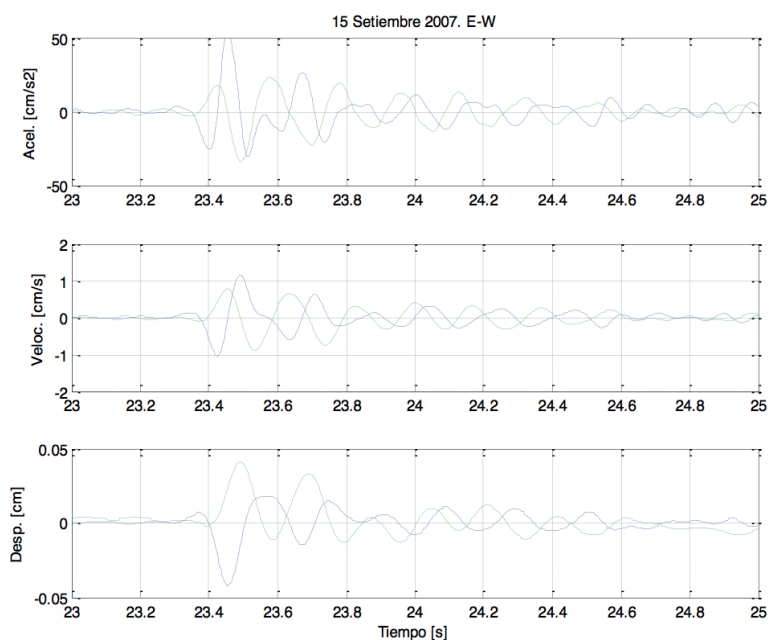

Figura 14. Aceleraciones, velocidades y desplazamiento registrados, a nivel de base (azul) y por encima del sistema de aislamiento (verde). Componente E-W, sismo del 15 Septiembre 2007. 

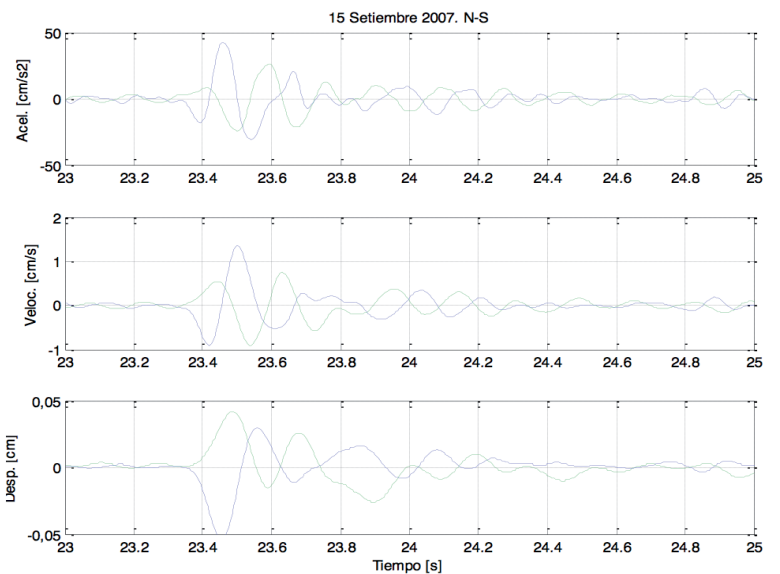

Figura 15. Aceleraciones, velocidades y desplazamiento registrados, a nivel de base (azul) y por encima del sistema de aislamiento (verde). Componente N-S, sismo del 15 Septiembre 2007.
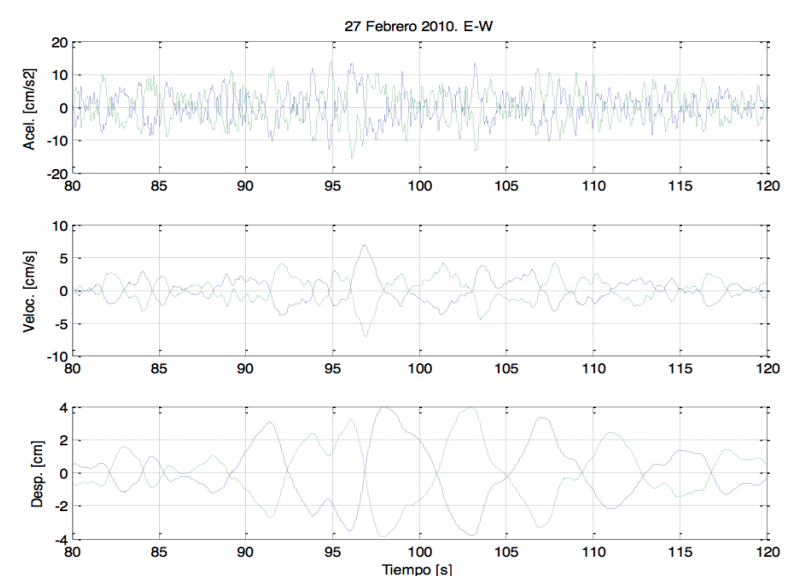

Figura 16. Aceleraciones, velocidades y desplazamiento registrados a nive de base (azul) y por encima del sistema de aislamiento (verde). Componente E-W, sismo del 22 Febrero 2010.
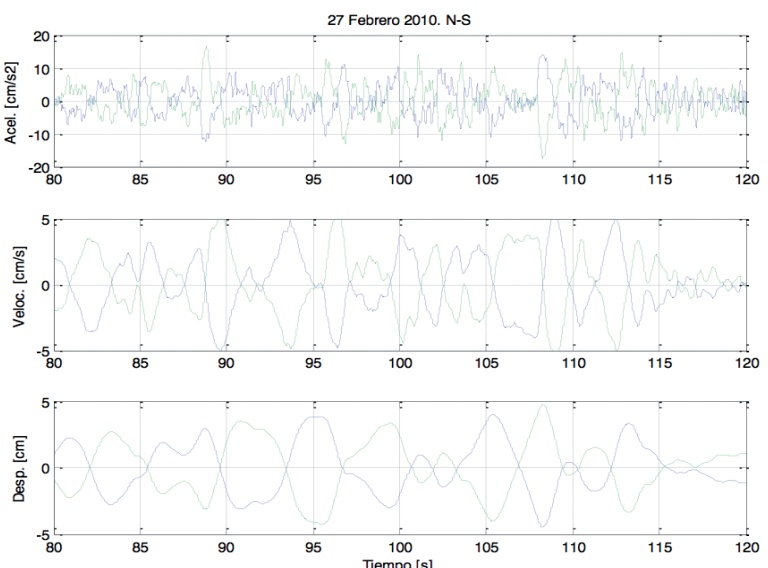

Figura 17. Aceleraciones, velocidades y desplazamiento registrados a nivel de base (azul) y por encima del sistema de aislamiento (verde). Componente $\mathrm{N}-\mathrm{S}$, sismo del 22 Febrero 2010.

Para los casos estudiados, de los gráficos se deduce, que las aceleraciones son atenuadas por el sistema de aislamiento. Esta atenuación es mayor cuando el registro de entrada es de mayor frecuencia caso que, corresponde a los sismos de origen local. Por otro lado la respuesta de aceleraciones no presenta un desfase constante para los sismos locales respecto de la señal de entrada; en tanto que para el caso del terremoto de Chile del 2010, se advierte un desfase prácticamente constante e igual a $180^{\circ}$.

En cuanto a la historia de las velocidades se puede afirmar que, por un lado disminuye el nivel de atenuación que proporciona el sistema de aislamiento y por otro se fortalece la constancia del desfase de la respuesta respecto de la señal de entrada. Nuevamente el terremoto de Chile del 2010 se destaca fuertemente en estos aspectos.

El análisis de los desplazamientos muestra en algunos casos atenuación y en otros, amplificación del movimiento por parte del sistema de aislamiento. Es en la historia de los desplazamientos donde se observa la mayor similitud entre la señal de entrada y la respuesta aunque con un desfasaje casi constante de $180^{\circ}$.

VALORES CALCULADOS VERSUS VALORES MEDIDOS A continuación se analizan los resultados obtenidos para las historias de tiempo de las aceleraciones, velocidades y desplazamientos según los modelos numéricos y se los compara con las aceleraciones provenientes de las mediciones instrumentales y con las velocidades y desplazamientos calculados por integración numérica. En las figuras 15 a 20 se muestran las gráficas de aceleración, velocidad y desplazamiento sobre el sistema de aislamiento en planta baja superponiendo los valores medidos a los valores obtenidos según el modelo tridimensional no lineal (MTNL) (Tornello y Sarrazin, 2012) y a los valores resultantes del modelo simple lineal (MSL).
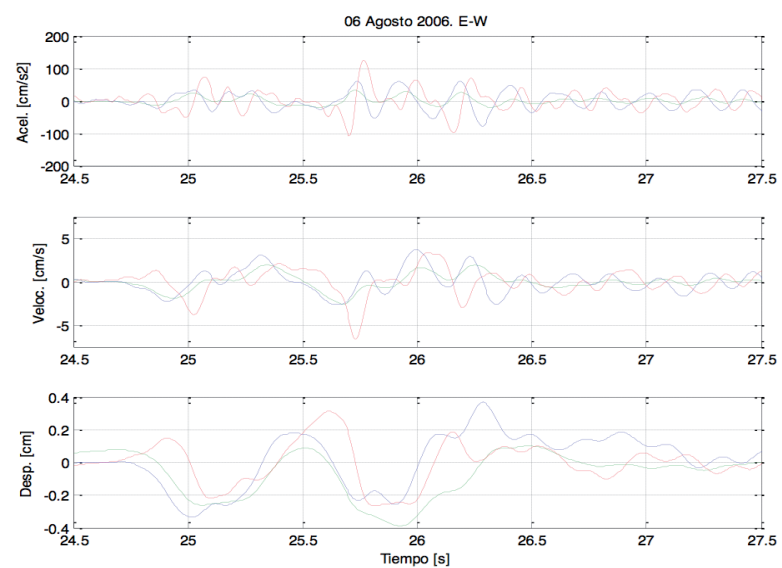

Figura 18. Comparación entre las aceleraciones, velocidades y desplazamiento registradas a nivel de planta baja, medida por acelerómetro (líneas azules) y la obtenida según MTNL (líneas verdes) y según el MSL (líneas rojas) para la componente horizontal E-W en el sismo del 6 de agosto de 2006. 

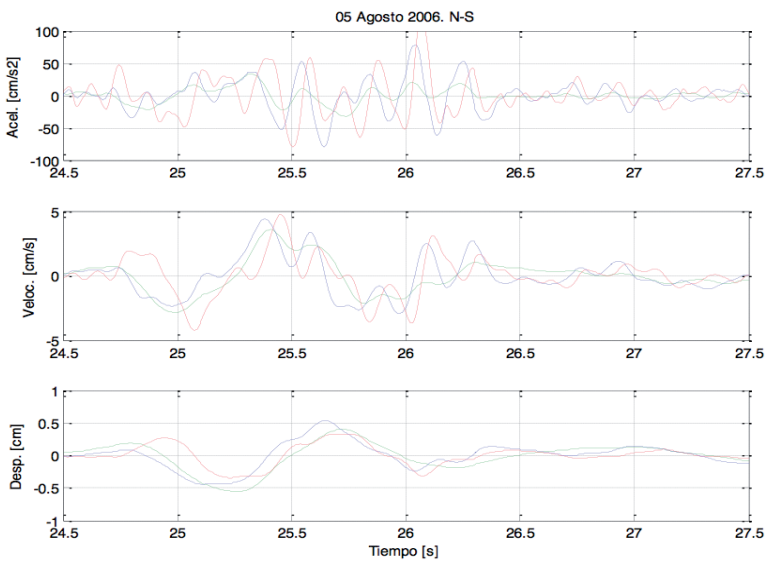

Figura 19. Comparación entre las aceleraciones, velocidades y desplazamiento registradas a nivel de planta baja, medida por acelerómetro (líneas azules) y la obtenida según MTNL (líneas verdes) y según el MSL (líneas rojas) para la componente horizontal N-S en el sismo del 06 de agosto de 2006.
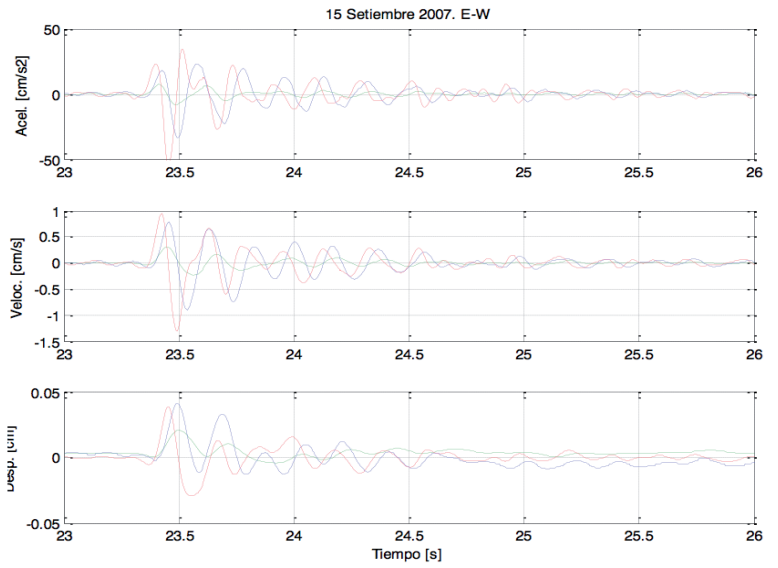

Figura 20. Comparación entre las aceleraciones, velocidades y desplazamiento registradas a nivel de planta baja, medida por acelerómetro (líneas azules) y la obtenida según MTNL (líneas verdes) y según el MSL (líneas rojas) para la componente horizontal E-W en el sismo del 15 setiembre de 2007.
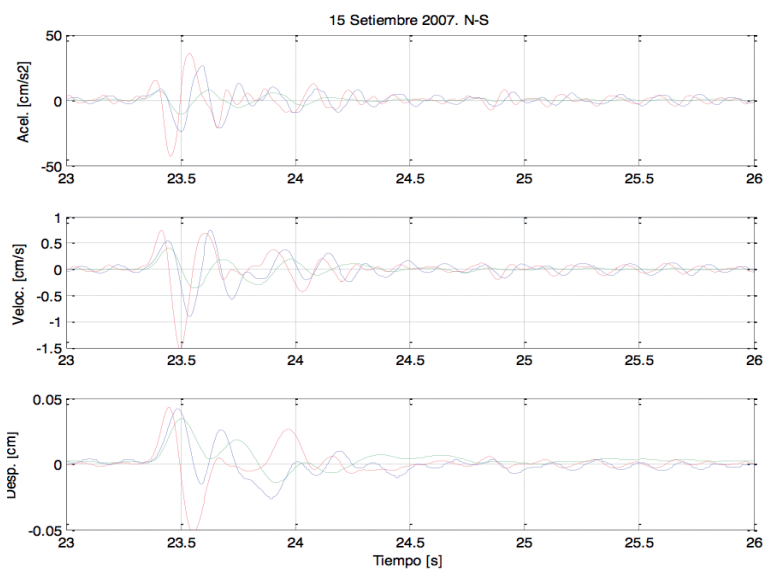

Figura 21. Comparación entre las aceleraciones, velocidades y desplazamiento registradas a nivel de planta baja, medida por de acelerómetro (líneas azules) y la obtenida según MTNL (líneas verdes) y según el MSL (líneas rojas) para la componente horizontal N-S en el sismo del 15 setiembre de 2007.
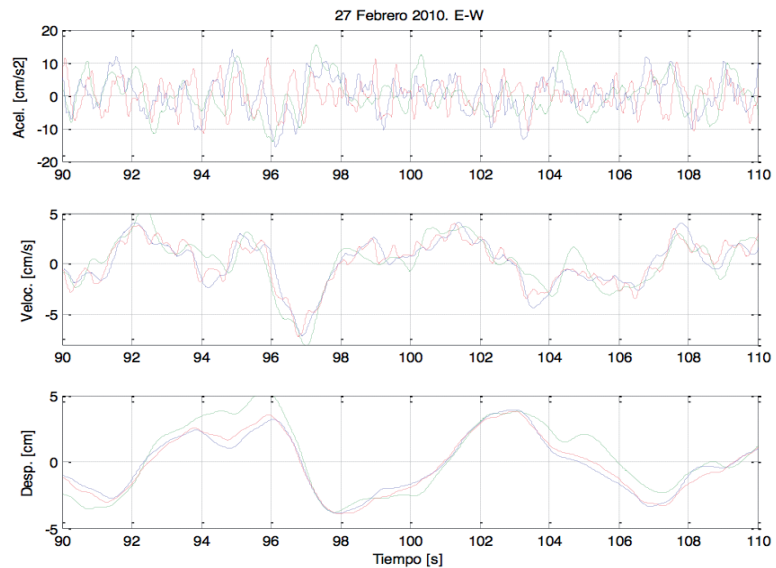

Figura 22. Comparación entre las aceleraciones, velocidades y desplazamiento registradas a nivel de planta baja, medida por acelerómetro (líneas azules) y la obtenida según el MTNL (líneas verdes) y según el MSL (líneas rojas) para la componente horizontal E-W en el sismo del 27 febrero de 2010.
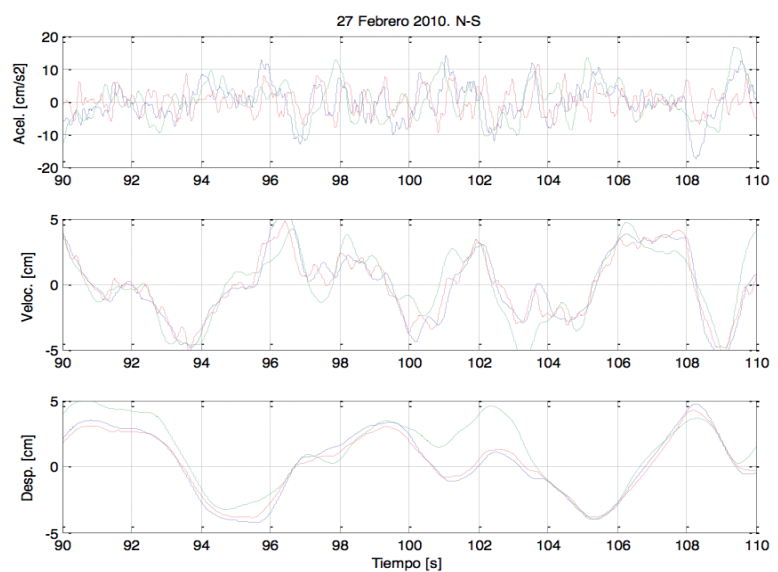

Figura 23. Comparación entre las aceleraciones, velocidades y desplazamiento registradas a nivel de planta baja medida por acelerómetro (líneas azules) y la obtenida según el MTNL (líneas verdes) y según el MSL (líneas rojas) para la componente horizontal $\mathrm{N}-\mathrm{S}$ en el sismo del 27 febrero de 2010.

Al analizar los resultados obtenidos en términos de aceleraciones se puede apreciar que, para los sismos locales, el MTNL acompaña, con buena aproximación, las ondas de aceleración medidas con desfases pequeños aunque presenta una subestimación en las amplitudes. El modelo simplificado (MSL) se aleja de los valores medidos, tanto en fase como en amplitudes, mostrando sobrevaloración de las aceleraciones. Caso especial es el sismo de foco lejano (Chile 2010) en donde tanto el MTNL como el MSL presentan aceptable aproximación en cuanto a la amplitud de los picos de aceleración pero con marcadas diferencias de fase.

En cuanto a las velocidades, para los sismos locales el MTNL acompaña bien la fase con cierta subestimación de los picos de cada onda. El MSL presenta importantes diferencias en fase y amplitudes. Para el caso particular del 
terremoto de Chile ambos modelos predicen adecuadamente la respuesta en velocidades.

Desde el punto de vista de los desplazamientos se aprecia que tanto el MTNL como el MSL aproximan bien la respuesta para los sismos del 6 de agosto de 2006 y el de Chile, de 2010. Se presume que para el caso del sismo del 15 de septiembre de 2007 las diferencias pueden ser causadas por los valores extremadamente reducidos de desplazamientos, $(0.4 \mathrm{~mm})$. Para el caso de sismos de baja frecuencia como el de Chile de 2010, el MSL arroja una muy buena aproximación.

CONCLUSIONES

Los resultados obtenidos del instrumental sísmico instalado en los edificio confirman la eficiencia de los dispositivos de aislamiento y su adecuado comportamiento frente a sismos sensibles.

Las reducciones de las respuestas en términos de aceleraciones del edificio con aislamiento sísmico en relación al edificio de base fija son significativas. En los casos mostrados en el presente trabajo los dispositivos de aislamiento han permitido reducir las aceleraciones entre tres a ocho veces los valores de aceleraciones registradas a nivel del techo del edificio de base tradicional.

Los resultados obtenidos de la instrumentación sísmica permitieron concluir que para

\section{REFERENCIAS BIBLIOGRÁFICAS}

Aguiar Falconi R., Tornello M. (2010). Análisis de respuestas de un edificio aislado con resortes de acero y amortiguadores viscosos. Revista Internacional de Ingeniería de Estructuras. Volumen 15, número 2, 2010. Pp. 1-47. ISSN 1390-0315. ESPE. Quito. Ecuador

Chopra A. K. (2014). Dinámica de Estructuras. 4o Edicion. Editorial Pearson. ISBN: 978-607-32-2239- 6. Pp 752

Frau, C. D. ySilva E. O. (2003). Demanda sísmica de aceleración y desplazamiento de grandes terremotos. VI Encuentro de Investigadores y Profesionales Argentinos de la Construcción (EIPAC) y XI Seminario Iberoamericano de Ingeniería Sísmica, Mendoza, Argentina.

Jangid R. S., Kelly J. M. (2001). Base Isolation for near-fault motions. Earthquake Engineering and Structural Dynamics. 30:691-707.

Kinemetrics inc. (2002). Altus Digital Recorder. User Manual

Martelli A. (2005). Modern Seismic Protection Systems for Civil and Industrial Structures. el edificio aislado estudiado, las aceleraciones son atenuadas por el sistema de aislamiento sin embargo, no se observa, el mismo fenómeno para las velocidades.

El modelo MTNL muestra un adecuado acompañamiento de la respuesta en la fase del movimiento (aceleración, velocidad y desplazamiento), aunque subestima las amplitudes de los picos de las aceleraciones y velocidades. Los desplazamientos, si bien presentan una pequeña atenuación, los valores encontrados se consideran aceptables.

El modelo MSL no presenta una buena aproximación para estimar las aceleraciones y velocidades sin embargo la aproximación mejora para los desplazamientos. Para el caso de sismos de baja frecuencia el modelo simplificado ajusta muy bien respecto de los valores medidos.

\section{AGRADECIMIENTOS}

El presente trabajo se ha desarrollado en el marco del proyecto de investigación 25/J091 y 25/ J088, financiado por la Universidad Tecnológica Nacional. Los autores agradecen a las autoridades de la Universidad por el financiamiento otorgado al proyecto y a las becas BINID asignadas al mismo. Se agradece a todos los docentes investigadores del CeReDeTeC, por los aportes y sugerencias realizadas al texto y al contenido del presente trabajo.

Congreso Chileno de Sismología e Ingeniería Antisísmica. IX Jornadas. Concepción. Chile. CD. ISSN 0718- 2678.

Naeim F.; Kelly J. M., (1999). "Design of Seismic Isolated Structures". Edit. John Wiley \& Sons, Inc. Printed in the United States of America.

Nawrotzki P (2001). Seismic Protection of structures by viscoelastic elements. The 8 th AsiaPacific Conference on Structural Engineering and Construction. Nanyang Technological University. Singapore.

Tornello M. Frau C., Palazzo C. (2013). Estructuras Edilicias con Dispositivos de Protección sísmica emplazadas en regiones de fallas activas. eduTecNe. Pp. 357. ISNB: 978-950-9064-73-7.

Tornello M (2012). Protección de estructuras con aislamiento sísmico de base. Una estrategia para reducir la vulnerabilidad sísmica de las obras civiles frente a terremotos destructivos. Edit. Academica Española. Schaltungsdienst Lange o:H G. Berlin, Books on Demand GMbH, Nordersdedt. Reha GmbH. ISBN: 978-3-84845168-5. 
Tornello M., Sarrazin M. (2012). Base Isolation building with high-damping spring system subjected to near fault earthquakes. Journal Earthquake and Structures 3 (3). Pp. 315-340.

Tornello M., Frau C. (2010). Experiencias sobre aislamiento sísmico en Mendoza, Argentina. Diseño, modelación y construcción. Revista Internacional de Ingeniería de Estructuras. Volumen 15, número 1, 2010. Pp. 1-47. ISSN 1390-0315. ESPE. Quito. Ecuador.

Tornello M., Frau C., Ciudad Real M., Gioacchini G., Panella S. (2010). Análisis comparativo entre respuesta instrumental y modelos numéricos de un edificio con aislamiento sísmico de base. Memorias del XXXIV Jornadas Sudamericanas de Ingeniería Estructural. San Juan Argentina. Tema 5. ID: 680.pdf. Pp. 1-15. Tornello M., Zarrazin M. (2007). Dynamic response of a building with base isolation for nearfault motion. 9th Canadian Conference on Earthquake Engineering. Paper 1057. Ottawa. CAEE, ACEP.

Wilson E. L. (2002). Three dimensional static and dynamic analysis of structures. A Physical approach with emphasis on Earthquake Engineering. CSI. Computer \& Structures Inc. SAP 90, SAP2000, SAFE, FLOOR and ETABS. 